\title{
Reduction of Metabolic Networks keeping Core Dynamics *
}

\author{
C. López Zazueta, ${ }^{*}$ O. Bernard, ${ }^{*}$ J.L. Gouzé. * \\ * Université Côte d'Azur, Inria, INRA, CNRS, UPMC Univ. Paris 06, \\ BIOCORE team, France (e-mail: claudia.lopez-zazueta@inria.fr, \\ olivier.bernard@inria.fr, jean-luc.gouze@inria.fr).
}

\begin{abstract}
Keywords: Metabolic engineering, Model reduction, Quasi Steady State Approximation,
\end{abstract} Tikhonov Theorem, Biological systems, Microalgae.

\section{INTRODUCTION}

Metabolic modeling has proved to be a very powerful tool to get a better insight into the metabolism of an organism. This approach has gained accuracy in the last decades, and turns out to be particularly efficient to improve production of target molecules, by understanding biological processes that influence metabolism. In particular, it allows to assess the main fluxes throughout a metabolic network (Baroukh et al., 2014; Nazaret and Mazat, 2008). But resulting models are of high dimension and difficult to use for control purpose.

For example, metabolic modeling has clarified production of triacylglycerols from microalgae and carbohydrates from cyanobacteria (Baroukh et al., 2015). Both compounds can then be turned into biofuel (biodiesel and bioethanol, respectively) with expected reduced environmental impacts (Lardon et al., 2009).

Metabolisms of microalgae and cyanobacteria are driven by the solar flux which supports fixation of $\mathrm{CO}_{2}$. Periodic fluctuation of light induces instationarity of their metabolisms, with accumulation of metabolites (especially lipids and carbohydrates). Therefore, such metabolisms are never at steady state.

However, most of the approaches dedicated to metabolism analysis assume balanced growth, i.e. Steady State Assumption (SSA), which leads to rough approximations. For instance, Flux Balance Analysis (FBA) (Orth et al., 2010) or Macroscopic Bioreaction Models (MBM) (Provost et al., 2006) are based on linear algebra to solve the equation $M \cdot V=0$, where $M$ is the stoichiometric matrix and $V$ is the vector of intracellular reaction rates.

Some approaches have attempted to introduce dynamics, for example, assuming that cell optimizes at each time instant an objective criterion using Dynamical Flux Balance Analysis (DFBA) (Mahadevan et al., 2002) or considering external conditions that might change continuously in a hybrid system, as proposed in Kaplan et al. (2009). But all of them address specific processes and a general mathematical framework has never been established.

\footnotetext{
* Claudia López Zazueta was supported by the National Council of Science and Technology of Mexico through the program CONACYTSECRETARÍA DE ENERGÍA-SUSTENTABILIDAD ENERGÉTICA 2015.
}

Furthermore, metabolic models are of high dimension, which makes their mathematical analysis and parameter identification complex. Identifying conditions to maximize productivity by a rigorous mathematical analysis is generally not possible.

Here we propose a method to reduce the dimension of a dynamical metabolic system, which is appropriate to derive model based control strategies. Contrary to nearly all existing works, the idea is to keep some dynamical components of the model, that are necessary especially when dealing with microalgae and cyanobacteria.

A first attempt in this direction was carried out with the DRUM method (Baroukh et al., 2014). This modeling approach considers subnetworks in Quasi Steady State (QSS), which are interconnected by metabolites that can accumulate. Then, Elementary Flux Modes (EFM) are computed in each subnetwork to reduce them using Quasi Steady State Assumption (QSSA). As result, the dynamics of accumulative metabolites form a reduced system of Ordinary Differential Equations (ODE).

DRUM approach has proven to provide sound results, with very efficient representation of accumulation of lipids and carbohydrates in microalgae submitted to light/dark cycles. However, as almost all methods developed for metabolic analysis, it relies on a series of assumptions whose mathematical bases have not been rigorously established. Beyond QSSA, which assumes "fast" and "slow" parts on the metabolism without delimiting them, these approaches also neglect intracellular dilution due to growth.

\section{RESULTS AND DISCUSSION}

The main objective of our work is to provide mathematical foundations for the reduction of metabolic networks to low dimensional dynamical models. In a first stage, we simplify the problem assuming linear kinetics in a metabolic network. For reducing the system accurately, we propose a dynamical approach that relies on time scale separation and the QSSA.

The reduction of a linear kinetic model, with a continuous input and a constant factor of dilution caused by growth, is analyzed through this method. The system is composed of subnetworks of fast reactions, which are connected by 
metabolites involved in slow dynamics (see Fig. 1). Hence, metabolites in subnetworks of fast reactions are assumed to be in QSS.

After a change of variables for the metabolites in QSS, the system of ODE corresponding to the metabolic model is presented as a slow-fast system:

$$
\begin{aligned}
\frac{d X}{d t} & =\bar{I}(t)-K_{1} \cdot X+K_{2} \cdot Y \\
\varepsilon \frac{d Y}{d t} & =K_{3} \cdot X-K_{4} \cdot Y,
\end{aligned}
$$

where $K_{4}$ is a strictly column diagonally dominant matrix (Horn and Johnson, 1985). In order to obtain a reduced dynamical model that approximates accurately its solution, we verify the conditions for applying Tikhonov Theorem for singularly perturbed systems (Kokotović et al., 1999).

Contrary to the classical assumption of Flux Balance Analysis (FBA) (where the whole system is assumed at steady state), our reduced model depends on a small system of differential equations, which represents the dynamics of the slow variables:

$$
\frac{d X}{d t}=\bar{I}(t)-K_{1} \cdot X+\left(K_{2} \cdot K_{4}^{-1} \cdot K_{3}\right) \cdot X .
$$

Moreover, dilution due to growth is not neglected in our method. Actually, keeping this dilution factor improves approximation precision and preserves qualitative (stability) features of the original system.

Once the reduced model is computed, it is possible to compare the concentration of the metabolites after an initial transition phase. Using the expression of the QSSA

$$
Y=K_{4}^{-1} \cdot K_{3} \cdot X,
$$

we mathematically prove that a metabolite in QSS has concentration one order of magnitude lower than any metabolite in the slow part of the system. This assertion is true subject to some flux constraints, which are also physically justified. For instance, not having traps for the flux from the input to the last metabolite in the chain is crucial.

Additionally, an algorithm to numerically estimate the parameters of the reduced system is proposed. We first use an identification strategy based on minimization, to estimate the parameters of the low dimensional ODE system obtained after the reduction. Then, we resolve a linear least squares problem for obtaining parameters required to approximate the concentration of metabolites in QSS.

Finally, we apply our method to a toy metabolic linear model and we compare the result with DRUM (Baroukh et al., 2015). The toy model includes a periodic input and reflects standard bricks in metabolic networks: combination of reversible and non-reversible reactions, with chains and cycles.

\section{ACKNOWLEDGEMENTS}

We acknowledge the support of the Inria Project Lab Algae in silico.

\section{REFERENCES}

Baroukh, C., Muñoz Tamayo, R., Steyer, J.P., and Bernard, O. (2014). DRUM: a new framework for

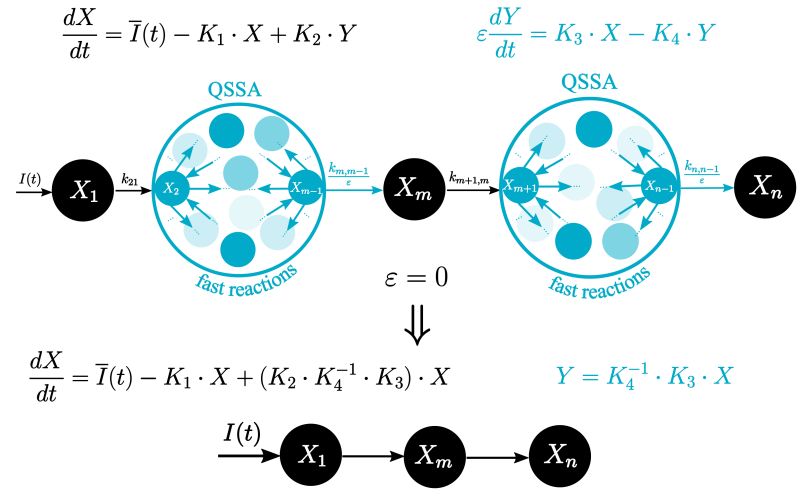

Fig. 1. Graphical abstract. Metabolic network with linear kinetics, one continuous input and constant factor of dilution because of growth. The slow part of the system is represented in black, with slow reactions and metabolites that reach the highest concentrations. The fast part is in blue, composed of fast reactions and metabolites in QSS with very low concentrations. A Quasi Steady State Reduction using Tikhonov Theorem leads to a low dimensional ODE system, which can be calibrated using minimization strategies.

metabolic modeling under non-balanced growth. application to the carbon metabolism of unicellular microalgae. PloS one, 9(8), e104499.

Baroukh, C., Muñoz Tamayo, R., Steyer, J.P., and Bernard, O. (2015). A state of the art of metabolic networks of unicellular microalgae and cyanobacteria for biofuel production. Metabolic Engineering, 30, 49-50.

Horn, R. and Johnson, C.R. (1985). Matrix Analysis. Cambridge University Press, New York.

Kaplan, U., Türkay, M., Biegler, L., and Karasözen, B. (2009). Modeling and simulation of metabolic networks for estimation of biomass accumulation parameters. Discrete Applied Mathematics, 157(10), 2483-2493.

Kokotović, P., Khalil, H.K., and O'reilly, J. (1999). Singular perturbation methods in control: analysis and design. Society for Industrial and Applied Mathematics, Philadelphia.

Lardon, L., Helias, A., Sialve, B., Steyer, J., and Bernard, O. (2009). Life-cycle assessment of biodiesel production from microalgae. Environmental sciences $\&$ technology, 43(17), 6475-6481.

Mahadevan, R., Edwards, J.S., and Doyle, F.J. (2002). Dynamic flux balance analysis of diauxic growth in Escherichia coli. Biophysical Journal, 83(3), 1331-1340.

Nazaret, C. and Mazat, J. (2008). An old paper revisited: "A mathematical model of carbohydrate energy metabolism. Interaction between glycolysis, the Krebs cycle and the H-transporting shuttles at varying ATPases load" by V. V. Dynnik, R. Heinrich and E. E. Sel'kov. Journal of theoretical biology, 252(3), 520-529.

Orth, J.D., Thiele, I., and Palsson, B.O. (2010). What is flux balance analysis? Nature biotechnology, 28(3), 245-248.

Provost, A., Bastin, G., Agathos, S.N., and Schneider, Y.J. (2006). Metabolic design of macroscopic bioreaction models: application to chinese hamster ovary cells. Bioprocess and biosystems engineering, 29(5-6), 349-366. 